\title{
Aufruf an alle Kolleginnen und Kollegen, die im Herbst für den Nationalrat oder den Ständerat kandidieren
}

Sehr geehrter Herr Kollege, sehr geehrte Frau Kollegin

Sie kandidieren im Herbst für den NR oder den SR? In diesem Fall möchten wir Ihnen die Gelegenheit geben, sich kurz in der Schweizerischen Ärztezeitung vorzustellen. Dazu bitten wir Sie, das untenstehende Formular auszufüllen und inkl. Photo bis am 25. August 2003 der Redaktion zukommen zu lassen.

Wir haben alle kantonalen Ärztegesellschaften und medizinischen Fachgesellschaften gebeten, diesen Brief an alle jene Kolleginnen und Kollegen weiterzuleiten, die für die Parlamentswahlen kandidieren.
Wir werden alle eingereichten Steckbriefe der Kandidatinnen und Kandidaten in alphabetischer Reihenfolge in der SÄZ Nr. 36 vom 3. September 2003 veröffentlichen. Wir hoffen, Ihnen eine attraktive Plattform und unseren Leserinnen und Lesern einen interessanten Einblick in die politische Vielfalt der Ärzteschaft zu vermitteln.

Für Rückfragen oder Kommentare stehen wir Ihnen gerne zur Verfügung.

Mit bestem Dank und freundlichen Grüssen Reto Steiner / Markus Trutmann

\begin{tabular}{l} 
Formular \\
Name, Vorname(n), Titel: \\
\hline Jahrgang: \\
Wohnort / Kanton: \\
Partei: \\
Facharzttitel: \\
Aktuelle berufliche Tätigkeit / Stellung: \\
Bisherige politische Tätigkeiten: \\
Geben Sie in dem nachfolgend zur Verfügung gestellten Raum Ihre wichtigsten politischen \\
Anliegen an (maximal 1000 Zeichen mit Leerzeichen).
\end{tabular}

Bitte ein farbiges oder schwarz-weisses Photo mitschicken!

\section{Redaktionsadresse}

Schweizerische Ärztezeitung, Steinentorstrasse 13, 4010 Basel, Tel. 0614678551 oder 03135911 50, E-Mail: redaktion.saez@emh.ch 


\section{Appel aux confrères et consœurs qui posent leur candidature à l'automne pour le Conseil national ou le Conseil des Etats}

Mesdames, Messieurs,

Vous êtes candidats pour le Conseil national ou le Conseil des Etats? Dans ce cas nous souhaitons vous donner la possibilité de vous présenter brièvement dans le Bulletin des médecins suisses. Vous voudrez bien, à cet effet, remplir la formule d'inscription ci-jointe, avec votre photo, et l'envoyer à la rédaction du BMS d'ici le 25 août 2003. Nous avons prié toutes les sociétés cantonales et les sociétés de discipline médicale de remettre cette lettre à leurs consœurs et confrères qui se portent candidats aux prochaines élections parlementaires.
Les identités des candidats par ordre alphabétique seront publiées dans le BMS $n^{\circ} 36$ du 3 septembre 2003. Nous espérons ainsi offrir un tremplin de choix pour les candidats et de donner à notre lectorat une approche intéressante de la diversité politique du corps médical.

Nous sommes volontiers à votre disposition pour toutes questions et commentaires.

En vous remerciant à l'avance, veuillez agréer, Mesdames, Messieurs, nos meilleures salutation.

Reto Steiner / Markus Trutmann

\begin{tabular}{l} 
Formule \\
Nom, prénoms, titres: \\
\hline Année de naissance: \\
\hline Lieu de résidence/canton: \\
\hline Parti: \\
Titre de spécialiste: \\
\hline Activité professionnelle actuelle / position: \\
\hline Antécédents politiques: \\
L'espace suivant est réservé aux principaux projets politiques qui vous tiennent à cœur \\
(1000 signes au maximum, espaces compris). \\
$\begin{array}{l}\text { Bulletin des médecins suisses, Steinentorstrasse 13, } 4010 \text { Bâle, tél. } 0614678551 \text { ou } 0313591150, \\
\text { e-mail: redaktion.saez@emh.ch }\end{array}$ \\
Veuillez y joindre une photographie en noir/blanc ou en couleurs! \\
\end{tabular}

